\title{
Why the Speed of Light (c) Keeps Constant?
}

\author{
Wei-Xing Xu \\ Newtech Monitoring Inc., Oshawa, Canada \\ Email: xweixing@hotmail.com
}

How to cite this paper: Xu, W.-X. (2017) Why the Speed of Light (c) Keeps Constant? Optics and Photonics Journal, 7, 67-73.

https://doi.org/10.4236/opj.2017.74006

Received: April 1, 2017

Accepted: April 25, 2017

Published: April 28, 2017

Copyright $\odot 2017$ by author and Scientific Research Publishing Inc. This work is licensed under the Creative Commons Attribution International License (CC BY 4.0).

http://creativecommons.org/licenses/by/4.0/ (c) (i) Open Access

\begin{abstract}
In this paper we demonstrate that the "pure" spacetime and electromagnetic spacetime are bound together. The "pure" spacetime and electromagnetic spacetime all behave as the wave in character and furthermore, both of them change at the same speed. Based on the understanding of the "pure" spacetime and electromagnetic spacetime, we give out the reasonable explanation why the speed of light keeps constant and how the gravity is created among the matters. In addition, some practical applications of the concepts developed in this work are proposed.
\end{abstract}

\section{Keywords}

Spacetime, Light Speed, Gravity, Photoelectronics, Light Communication, Electromagnetic Wave

\section{Introduction}

In the process of understanding the universe, the speed of light is a puzzle for human being to explain, that is, it doesn't matter whether the light source is moving toward or away from us, we got the same speed of light [1] [2]. When Einstein setup the relativity theory, he supposed the speed of light is constant and furthermore, nobody in our universe can go faster than the light. Einstein didn't give the reason why the speed of light is constant, therefore, till now we still can't understand how and why it is.

In the relativity theory, Einstein told us the curved space and inflation of time [3]. If the space is really curved and time is inflated, the ratio of space over time must keep constant. Only under this condition, there exists the possibility for the speed of light to keep as a constant. Based on the relativity theory, the earth doesn't turn around the sun but just follows the curved spacetime which is formed by the earth and sun. If it is true, why the light passing the sun does not follow the curved spacetime formed by the earth and the sun?

All these questions make scientists explore the deep meaning of our universe 
or spacetime. As a first step in this effort, we have to go back to the beginning of the question: why the speed of light keeps constant?

\section{Theory}

In order to understand why the speed of light is constant, we have to make clear what is spacetime? The spacetime is the universe around us, which includes $\mathrm{x}, \mathrm{y}$, $z$, three space dimensions and $t$, one time dimension. Then, the following question arises: does the spacetime change? Based on the astronomy observation, the spacetime not only changes but also changes in accelerating [4] [5] [6]. Therefore, we can suppose $\Psi(\mathrm{x}, \mathrm{y}, \mathrm{z},-i t)$ to represent the spacetime (We can call "pure spacetime". The more familiar words to publics are "empty" and "vacuum", but we prefer to use "pure spacetime" here and following.).

$$
\Psi(x, y, z,-i t)=0
$$

Then,

$$
\frac{\partial^{2} \Psi}{\partial x^{2}} \mathrm{~d}^{2} x+\frac{\partial^{2} \Psi}{\partial y^{2}} \mathrm{~d}^{2} y+\frac{\partial^{2} \Psi}{\partial z^{2}} \mathrm{~d}^{2} z=\frac{\partial^{2} \Psi}{d t^{2}} \mathrm{~d}^{2} t
$$

Supposing our universe is homogeneous in all direction [7], then, we have

$$
\frac{\partial^{2} \Psi}{\partial x^{2}} \mathrm{~d}^{2} x=\frac{\partial^{2} \Psi}{\partial y^{2}} \mathrm{~d}^{2} y=\frac{\partial^{2} \Psi}{\partial \mathrm{z}^{2}} \mathrm{~d}^{2} z
$$

And using L to represent all the space dimensions,

Then,

$$
3 \frac{\partial^{2} \Psi}{\partial L^{2}} d^{2} L=\frac{\partial^{2} \Psi}{\partial t^{2}}
$$

This result tells us that any point in our spacetime can be described by Eq. (4). Making rearrangement of Eq. (4), we obtain

$$
\frac{\partial^{2} \Psi}{\partial L^{2}}=\frac{1}{3 \frac{d^{2} L}{d^{2} t}} \frac{\partial^{2} \Psi}{\partial t^{2}}
$$
have

By defining $3 \frac{\mathrm{d}^{2} L}{\mathrm{~d} t^{2}}=c^{2}$, where $\mathrm{c}$ is the speed of spacetime variation, then, we

$$
\frac{\partial^{2} \Psi}{\partial L^{2}}=\frac{1}{c^{2}} \frac{\partial^{2} \Psi}{\partial t^{2}}
$$

In fact, Eq. (6) is the same as the wave equation, where the parameter $\mathrm{c}$ corresponds to the wave propagation speed [8]. This means that any point in the spacetime behaves as a wave, which we can call the spacetime wave.

Now we consider the variation of electric and magnetic fields with time for light.

Fortunately, Maxwell already did the work one hundred years ago. We can directly use his result here [9] [10]

$$
\begin{aligned}
& \frac{\partial^{2} E}{\partial L^{\prime 2}}=\frac{1}{c^{\prime 2}} \frac{\partial^{2} E}{\partial t^{\prime 2}} \\
& \frac{\partial^{2} B}{\partial L^{\prime 2}}=\frac{1}{c^{\prime 2}} \frac{\partial^{2} B}{\partial t^{\prime 2}}
\end{aligned}
$$


Where $c^{\prime}$ is the electromagnetic wave propagation speed. Comparing Eq. (6) with Eq. (7) and Eq. (8), we found they all keep the same formulation as standard wave equation. Making the rearrangements of Eq. (6), Eq. (7) and Eq. (8), we got

$$
\begin{gathered}
c=\left[\frac{\frac{\partial^{2} \Psi}{\partial t^{2}}}{\frac{\partial^{2} \Psi}{\partial L^{2}}}\right]^{1 / 2} \\
c^{\prime}=\left[\frac{\frac{\partial^{2} \mathrm{E}}{\partial t^{2}}}{\frac{\partial^{2} \mathrm{E}}{\partial L^{2}}}\right]^{1 / 2} \\
c^{\prime}=\left[\frac{\frac{\partial^{2} \mathrm{~B}}{\partial t^{2}}}{\frac{\partial^{2} \mathrm{~B}}{\partial L^{2}}}\right]^{1 / 2}
\end{gathered}
$$

Then, we have

$$
c-c^{\prime}=\left[\frac{\frac{\partial^{2} \Psi}{\partial t^{2}}}{\frac{\partial^{2} \Psi}{\partial L^{2}}}\right]^{1 / 2}-\left[\frac{\frac{\partial^{2} \mathrm{E}}{\partial t^{2}}}{\frac{\partial^{2} \mathrm{E}}{\partial L^{2}}}\right]^{1 / 2} \text { or } c-c^{\prime}=\left[\frac{\frac{\partial^{2} \Psi}{\partial t^{2}}}{\frac{\partial^{2} \Psi}{\partial L^{2}}}\right]^{1 / 2}-\left[\frac{\frac{\partial^{2} \mathrm{~B}}{\partial t^{2}}}{\frac{\partial^{2} \mathrm{~B}}{\partial L^{2}}}\right]^{1 / 2}
$$

For Eq. (12), there are three cases.

1) $c-c=0$. That means the electromagnetic wave speed is the same as that of "pure" spacetime we lived in, then, we can't independently determine the speed of light because we can't find independent reference for this measurement. That is why we always get the same speed of light which is irrelative to whether the light source is moving or not. If we hope to measure the absolute speed of light, we have to stay outside the electromagnetic spacetime. To do so, we have to move faster than the electromagnetic wave to get out of the electromagnetic spacetime, that means $c-c<0$, this conflicts with the assumption of $c-c=0$, therefore, it is impossible for human being to get outside the electromagnetic spacetime, at least at present. That is why we always get the same speed of light. One may ask why we get the current constant as the speed of light instead of others. At present, we still don't know the definitive answer for this question yet. One possible reason is the current constant is determined by the spacetime which reflect some symmetry of spacetime, just like the translation symmetry in crystal. Why? The constant of speed of light not only appeared in the astronomy dimension (for example, we use light year as unit of distance in astronomy) but also appeared in the fine structure constant for atom [11] [12]. Another question for us to think about is if we can stay outside the electromagnetic spacetime, which means we can find the reference which is independent of the electromagnetic spacetime, does the speed of light change? At present, we still can't give certainty answer about this question. But some hints, such as the spacetime expansion (especially our spacetime experienced the fast expansion at the begin- 
ning), tell us that the absolute speed of light is changing, not a constant because the electromagnetic spacetime keeps the same speed as the "pure" spacetime [4].

Some scientists figure out the "ether" existing everywhere to support the light to propagate, but till now, all experiments give negative answer for the existence of the "ether" [13]. Based on our discussion above, the electromagnetic spacetime changes with the "pure" sapcetime at the same speed $(c-c=0)$, that means the electromagnetic spacetime is in statics relative to the "pure" spacetime, therefore, the light doesn't need some medium to support its propagation because the electromagnetic spacetime relative to the "pure" spacetime doesn't move at all $(c-c=0)$.

2) $c-c>0$. Following the Newton mechanics, if we ignore the difference between $\mathrm{dt}$ for "pure" spacetime and dt' for electromagnetic spacetime, then we get

$$
\mathrm{c}-\mathrm{c}^{\prime}=\frac{F}{m} \mathrm{~d} t
$$

where $\mathrm{F}$ is the force resulting from the interaction between the electromagnetic spacetime and "pure" spacetime, which is an attractive force among the photon; $\mathrm{m}$ is the mass of the photon. In literature, some scientists propose the photon has static mass but is still not accepted generally yet. If photon does have static mass, then we should observe the attractive force between photons, and this attractive force among photons will make the photons self focusing, then all photons will focus together that will be terrible situation due to the huge energy concentrated at one point. Fortunately, this situation never occurs till now.

It is interesting if we think this result reversely, that is, if the matter has static mass, then the attractive force should exist among matters, which may be the origin of the gravity of our universe. In fact, for the spacetime we lived in, all in this electromagnetic spacetime are electromagnetic in character, including the author of this work. Therefore, we can imagine all matter in our electromagnetic spacetime is the compressed or concentrated electromagnetic spacetime (this means that the "pure" spacetime also can be compressed to create the matter). From Eq. (13), among the compressed electromagnetic spacetime or matter, there exists attractive force, which is called gravity. Furthermore, this attractive force exists generally among the compressed electromagnetic spacetime or matters and always attractive in character, which means the gravity comes from the relative accelerating movement of matter to the "pure" spacetime. This situation can be illustrated in Figure 1.

Here we hope to develop some practical application from the discussion above. We know the speeds of light are different for light passing the compressed spacetime or matter and vacuum or "pure" spacetime. Then, Eq. (14) can be used to determine the mass of the compressed spacetime or matter.

$$
\text { Mass }=\sum k_{i} s_{i} \mathrm{~d} t_{i}
$$

where $k_{\mathrm{i}}$ is the constant which is different for the different path of light passing the matter, $s_{\mathrm{i}}$ is the surface area of the matter which the light pass through, $\mathrm{dt}_{\mathrm{i}}$ is the time for light passing through the matter. We take a simple case as an example for application of Eq. (14) (Figure 2). 


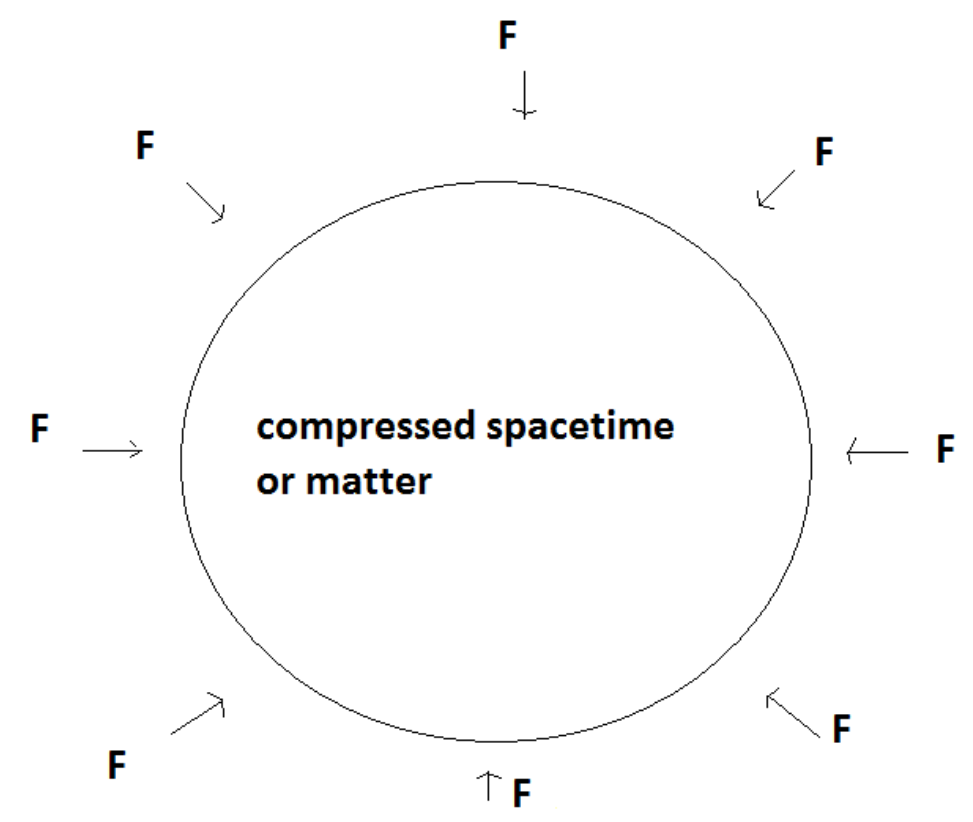

Figure 1. The illustration of the origin of the gravity among matters.
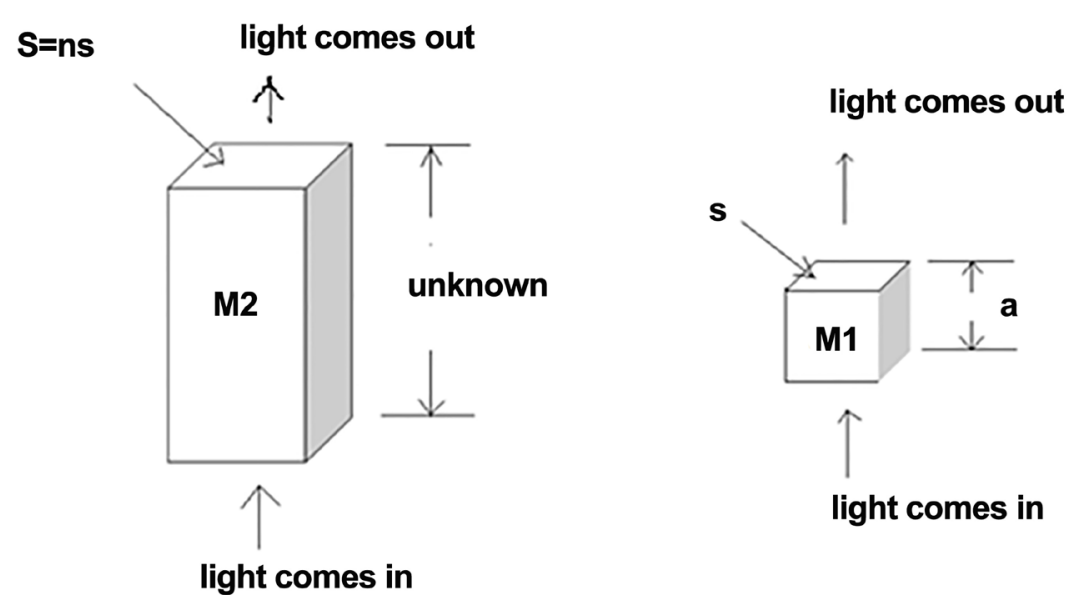

Figure 2. The illustration for how to determine the mass of matter by light measuring experiment.

where $m_{1}$ is known mass of the matter, $\mathrm{M}_{2}$ is unknown mass of the matter and $\mathrm{S}$ $=n s$.

Then, $k s=\frac{m_{1}}{\Delta t_{1}}=\frac{m_{1} c^{\prime}}{a}$, we get $M_{2}=\frac{n m_{1} c^{\prime} \Delta t_{2}}{a}$. In general, we also can use the Eq. (14) to diagnose the crystal perfectness of matter or homogeneity of matter.

3) $c-c<0$. This situation corresponds to the fact that the electromagnetic spacetime change faster than the "pure" spacetime. Then, F in Eq. (13) will become repelling force instead of the attractive force among photons discussed in case 2 above. Maybe this is the reason causing the continuous expansion of our universe. Alternatively, m may take negative sign which also cause the $c-c<0$. 
In this case, The $\mathrm{F}$ is still attractive in character. Till now, the physical meaning of negative mass is still not clear, for instance, is it an unknown form of matter or antimatter? If the negative mass means the antimatter, then, the attractive force $(F)$ means that the gravity also exists among the antimatter. As to whether the gravity constant $(\mathrm{G})$ is the same as normal matter or not, it is not clear and need more experiment to verify.

Based on the Eq. (12), we discussed three situations, that is, the case 1, 2, 3 above. For case 2 and 3, the photon must have static mass. Before we got solid evidence for the existence of the static mass of photon, we have to accept the case 1 as the real and unique situation around us. However, some discussions for case 2 and 3 definitively can be applied to the matter with static mass.

Till now, all the physical laws or theories are made in the electromagnetic spacetime, we have to follow those laws without choice.

\section{Conclusion.}

From the discussion above, we know that our electromagnetic spacetime keeps the same speed of variation with the "pure" spacetime. All matters in electromagnetic spacetime can't move faster than the electromagnetic wave because nothing can be faster than the "pure" spacetime. The absolute speed of light is determined by the nature, not by the human being. We can't determine the absolute speed of light because we can't find the independent reference to conduct the measurement. The absolute speed of light may change because there are some evidences showing our spacetime experienced fast expansion at the beginning [14]. If this is true, then, the absolute light speed should change with the development of our spacetime. However, this variation of the absolute speed of light has nothing to do with us because we can't stay outside of the electromagnetic spacetime.

\section{References}

[1] Michelson, A.A. (1878) Experimental Determination of the Velocity of Light. Proceedings of the American Association of Advanced Science, 27, 71-77.

[2] Einstein, A. (1905) Zur Elektrodynamikbewegter Koerper. Annalen der Phyisk, 17, 890-921. (In German)

[3] Einstein, A. (1915) Die Feldgleichungen der Gravitation. Sitzungsberichteder Preussischen Akademie der Wissenschaftenzu, Berlin, 844-847.

[4] Simon, Singh; (2004) Big Bang. An Division of Harper Collins Publishers, Fourth Estate.

[5] Peebles, P.J.E. and Ratra, B. (2003) The Cosmological Constant and Dark Energy. Reviews of Modern Physics, 75, 559-606. https://doi.org/10.1103/RevModPhys.75.559

[6] Pain, R. and Astier, P. (2014) Observational Evidence of the Accelerated Expansion of the Universe. Comptes Rendus Physique, 13, 521-538.

[7] Andrew, L. (2003) An Introduction to Modern Cosmology. 2nd Edition, John Wiley \& Sons, Hoboken, New Jersey.

[8] Speiser, D. (2008) Discovering the Principles of Mechanics, 1600-1800. Birkhaeuser, 
Basel, 191

[9] Jackson, J.D. (1998) Classical Electrodynamics. 3rd Edition, Wiley, Hoboken, New Jersey.

[10] Maxwell, J.C. (1865) A Dynamical Theory of the Electromagnetic Field. Philosophical Transactions of Royal Society of London, 155, 459-512. https://doi.org/10.1098/rstl.1865.0008

[11] Aoyama, T., Hayakawa, M., Kinoshita, T. and Nio, M. (2012) Tenth-Order QED Contribution to the Electron g-2 and an Improved Value of the Fine Structure Constant. Physical Review Letters, 109, 111807. https://doi.org/10.1103/PhysRevLett.109.111807

[12] Uzan, J.-P. and Leclercq, B. (2008) The Natural Laws of the Universe: Understanding Fundamental Constants. Springer, Berlin, 43-44. https://doi.org/10.1007/978-0-387-74081-2

[13] Michelson, A.A. and Morley, E.W. (1887) On the Relative Motion of the Earth and the Luminiferous Ether. American Journal of Science, 34, 333-345. https://doi.org/10.2475/ajs.s3-34.203.333

[14] Guth, A.H., Kaiser, D.I. and Nomura, Y. (2014) Inflationary Paradigm after Planck 2013. Physics Letters B, 733, 112-119. https://doi.org/10.1016/j.physletb.2014.03.020

Submit or recommend next manuscript to SCIRP and we will provide best service for you:

Accepting pre-submission inquiries through Email, Facebook, LinkedIn, Twitter, etc. A wide selection of journals (inclusive of 9 subjects, more than 200 journals) Providing 24-hour high-quality service User-friendly online submission system Fair and swift peer-review system Efficient typesetting and proofreading procedure Display of the result of downloads and visits, as well as the number of cited articles Maximum dissemination of your research work

Submit your manuscript at: http://papersubmission.scirp.org/ Or contact opj@scirp.org 